\title{
Epigenetic impairment and blunted transcriptional response to Mycobacterium tuberculosis of alveolar macrophages from persons living with HIV
}

Wilian Correa-Macedo ${ }^{1,2,3 \ddagger}$, Vinicius M. Fava ${ }^{2,3 \ddagger}$, Marianna Orlova ${ }^{2,3}$, Pauline Cassart ${ }^{2,3}$, Ron Olivenstein $^{4}$, Joaquín Sanz ${ }^{5}$, Anne Dumaine ${ }^{6}$, Renata H.M. Sindeaux ${ }^{7}$, Vania Yotova ${ }^{7}$, Alain Pacis $^{8}$, Josée Girouard ${ }^{2,9}$, Barbara Kalsdorf ${ }^{10,11}$, Christoph Lange ${ }^{10,11}$, Jean-Pierre Routy ${ }^{2,9}$, Luis B. Barreiro ${ }^{6 \dagger}$, Erwin Schurr ${ }^{1,2,3 \dagger^{*}}$.

${ }^{1}$ Department of Biochemistry, Faculty of Medicine, McGill University, Montréal, QC, Canada.

${ }^{2}$ Program in Infectious Diseases and Global Health, The Research Institute of the McGill University Health Centre, Montréal, QC, Canada.

${ }^{3}$ McGill International TB Centre, Department of Medicine, Faculty of Medicine, McGill University, Montréal, QC, Canada.

${ }^{4}$ Translational Research in Respiratory Diseases Program, The Research Institute of the McGill University Health Centre, QC, Montréal, Canada.

${ }^{5}$ Institute for Bio-computation and Physics of Complex Systems BIFI, Department of Theoretical Physics, University of Zaragoza, Zaragoza, Spain.

${ }^{6}$ Department of Medicine, Genetic Section, University of Chicago, Chicago, IL, USA.

${ }^{7}$ CHU Sainte-Justine Hospital Research Centre, Montréal, QC, Canada.

${ }^{8}$ Canadian Centre for Computational Genomics, McGill University and Genome Quebec Innovation Center, Montréal, QC, Canada.

${ }^{9}$ Chronic Viral Illnesses Service and Division of Hematology, McGill University Health Centre, Montréal, QC, Canada.

${ }^{10}$ Division of Clinical Infectious Diseases, Research Center Borstel, Borstel, Germany.

${ }^{11}$ International Health/Infectious Diseases, University of Lübeck, German Center for Infection Research (DZIF), Partner Site Hamburg-Lübeck-Borstel, Lübeck, Germany.

+ Share first authorship

$\uparrow$ Share senior authorship

* Corresponding author: erwin.schurr@mcill.ca 


\begin{abstract}
Persons living with HIV (PLWH) are at increased risk of tuberculosis (TB). HIV-associated TB is often the result of recent infection with Mycobacterium tuberculosis (Mtb) followed by rapid progression to disease. Alveolar macrophages (AM) are the first cells of the innate immune system that engage $M t b$, but how HIV and antiretroviral therapy (ART) impact on the antimycobacterial response of AM is not known. To investigate the impact of HIV and ART on the transcriptomic and epigenetic response of AM to $M t b$, we obtained AM by bronchoalveolar lavage from 20 PLWH receiving ART, 16 control subjects who were HIV-free (HC), and 14 subjects who received ART as pre-exposure prophylaxis (PrEP) to prevent HIV infection. Following in-vitro challenge with $M t b$, AM from each group displayed overlapping but distinct profiles of significantly up- and down-regulated genes in response to $M t b$. Compared to HC subjects, AM isolated from PLWH and PrEP subjects presented a substantially weaker transcriptional response. Further investigation of chromatin structure revealed that AM from control subjects challenged with $M t b$ responded with pronounced accessibility changes in over ten thousand regions. In stark contrast, AM obtained from PLWH and PrEP subjects displayed no significant changes in their chromatin state in response to $M t b$. Collectively, these results revealed a previously unknown adverse effect of ART on the epigenetic landscape and transcriptional responsiveness of AM.
\end{abstract}




\section{Introduction}

Tuberculosis (TB) is globally the leading cause of death due to a single pathogen. In 2018, there were an estimated 1.45 million deaths caused by TB, which included 250,000 people living with the human immunodeficiency virus (HIV) (1). Of the estimated 10 million people who fell ill with TB, 850,000 were persons living with HIV (PLWH) indicating a 2.2-fold increased risk of death due to TB in HIV-positive relative to HIV-negative persons. Globally, the proportion of notified HIV-positive TB cases on antiretroviral therapy was $86 \%$ with large gaps among countries (1). However, even people on long-term antiretroviral therapy (ART) still have significantly increased risk of developing TB (2), which is usually the result of new infection rather than reactivation of latent $\mathrm{TB}(3-6)$.

Transmission of Mycobacterium tuberculosis (Mtb) occurs by aerosols that are inhaled by an exposed person. Results from the mouse model show that after reaching the lung alveoli, $M t b$ bacilli are being rapidly taken up by alveolar macrophages (AM). Following a delay of approximately 10 days, successful establishment of infection occurs once $M t b$-infected AM traverse the airway epithelium and establish themselves in the lung interstitium. There, transfer of $M t b$ to permissive inflammatory macrophages occurs and T-cell priming is initiated in the draining lymph nodes (7). However, much of the AM-Mtb interaction in the alveoli remains unknown.

In humans, not each exposure results in successful infection, and a subset of highly exposed persons entirely resist infection as inferred from the absence of $\mathrm{CD}^{+} \mathrm{T}$ cell anti-Mtb immunity $(8,9)$. Hence it seems possible that human AM are capable to limit transfer of inhaled Mtb bacilli to the lung interstitium. The increased risk of $M t b$ infection and TB disease for PLWH may 
therefore reflect an impaired ability of AM to restrict $M t b$ to the alveoli, thus placing early events in the $M t b$-host interaction as a central aspect of TB resistance.

Based on the above considerations, we decided to investigate the transcriptome and epigenome of the ex-vivo AM-Mtb interaction employing AM obtained from PLWH and HIV-negative participants. Since ART is given to most PLWH, we also included an additional control group consisting of persons who are HIV-negative receiving ART for pre-exposure prophylaxis (PrEP).

\section{Results}

\section{Study subjects}

To investigate the influence of HIV on the ability of AM to mount a response to Mtb infection, we studied three groups of subjects: PLWH receiving ART $(n=20)$, persons without HIV but receiving ART as pre-exposure prophylaxis (PrEP; $n=14$ ) and persons without HIV not receiving PrEP (healthy controls, HC; $\mathrm{n}=16$, Table 1). We obtained AM by BAL from all subjects and challenged the cells in vitro with $M t b$. RNA and DNA were isolated from uninfected and $M t b$ challenged cells and used to assess gene expression levels by RNA-seq, chromatin structure by ATAC-seq, and presence of the histone activation mark H3K27ac by ChIP-seq (Fig. 1A).

However, due to different quality-control procedures and variable numbers of AM obtained per subject, different counts of subjects were used for the transcriptomics and epigenetics experiments (Fig. 1A). 
medRxiv preprint doi: https://doi.org/10.1101/2021.01.26.21250318; this version posted January 29, 2021. The copyright holder for this preprint (which was not certified by peer review) is the author/funder, who has granted medRxiv a license to display the preprint in perpetuity.

It is made available under a CC-BY-NC-ND 4.0 International license .

Alveolar macrophages from PLWH and PrEP subjects display blunted mRNA transcriptomic changes in response to Mtb

Across the three phenotypic groups, we identified 1,626 differentially expressed genes (DEG) in response to Mtb (Data File S1). However, there were pronounced DEG count differences in their transcriptional responses (Fig. 1B). AM from HC subjects showed 1,434 DEG in response to Mtb as compared to only 679 and 255 DEG in PLWH and PrEP subjects, respectively (Fig. 1B and Fig. S1A). The magnitude of AM transcriptional response to $M t b$ also differed among the three groups. The smallest mean absolute $\log 2$ fold change $(\log 2 \mathrm{FC})$ in response to $M t b$ infection was observed among PrEP subjects while HC subjects displayed the strongest transcriptional response. (Fig. 1C and Fig. S1A). To test if Mtb effects in PLWH and PrEP subjects were weaker for all DEGs or only for specific gene sets, we assessed the correlations of $M t b$-triggered $\log \mathrm{FC}$ across the three groups. We observed that $\log 2 \mathrm{FC}$ values were strongly correlated among all three groups, but consistently higher for corresponding genes from HC subjects (Fig. S1B) which suggested a general transcriptional impairment in cells from PLWH and PrEP subjects. Pathway and GO-term enrichment analysis revealed that $M t b$-induced transcriptional changes were enriched in biological functions representative of macrophage anti-mycobacterial host responses, such as interferon (IFN) and Toll-like receptor signalling pathways (Fig. 1D). However, compared to HC subjects, the number of significant GO-terms/pathways was substantially lower for the PLWH and PrEP groups (Table S1). Moreover, even among shared significant terms, the cumulative transcriptional response of genes in these terms was stronger in the HC group, which further emphasized the blunted anti-mycobacterial response by PLWH and PrEP subjects (Fig. 1D). We also tested which genes had a significantly different magnitude of response to $M t b$ among phenotype groups (Fig. 1E and Data File S1). We identified 333 genes 
significantly different between the groups (Data File S2) with the majority displaying lower upregulation in response to $M t b$ for PLWH and PrEP groups relative to the HC group (Fig. S1, C and D and Table S1). These results provided a formal confirmation that the transcriptional response to $M t b$ challenge by $\mathrm{AM}$ of $\mathrm{HC}$ was more vigorous compared to the one mounted by PrEP and PLWH subjects.

To explore if the group differences were mainly driven by strong outliers, we derived per subject average $\log 2 \mathrm{FC}$ across DEG in significant pathways/GO-terms. We found that AM from HC subjects displayed a more pronounced group transcriptional response to $M t b$. For example, the mean $\log 2 \mathrm{FC}$ for the term "I-kappaB kinase/NF-kappaB signaling” was 3.22-fold $\left(p=2.4 \times 10^{-3}\right)$ and 2.39-fold $\left(p=3.8 \times 10^{-2}\right)$ higher in HC as compared to PLWH and PrEP, respectively (Fig. 1F). GO-terms and pathways tagged by the DEG from PLWH and PrEP vs HC, which were significantly more induced in AM from HC subjects, revealed a predominance of innate-immune and intracellular defense response terms (Fig. 1G). This observation alongside the exclusion of possible outlier-driven group differences supported the reduced ability of AM from PLWH and PrEP subjects to mount an effective anti-mycobacterial transcriptional response to challenge with Mtb.

Mtb does not induce chromatin remodelling in AM from PLWH and PrEP subjects

Next, we evaluated $M t b$-triggered epigenetic changes of AM chromatin accessibility and H3K27 acetylation. Analysis of Differentially Open Chromatin (DOC) in response to Mtb indicated that AM from $\mathrm{HC}$ had significant increased accessibility (opening) in 8,389 regions and repression (closing) in 3,971 regions (Fig. 2A; Data File S3). In contrast, AM isolated from PLWH or PrEP 
subjects displayed near complete absence of significant chromatin remodeling in response to $M t b$

(Fig. 2, A and B). A broad-based epigenetic impairment was confirmed for AM from PLWH subjects by tracking regions with differently H3K27 acetylation (DAc), a mark of active chromatin (Fig. 3A; Data File S4). Pathway and GO-term enrichment analyses of genes annotated to DOC and DAc in HC identified typical viral and bacterial response terms (Fig. 2C; Fig. 3B). As in the transcriptomics analyses, the epigenetic response to $M t b$ by AM from HC strongly implicated host immune response to pathogens and immune-related signalling pathways, which was mainly driven by opening DOCs and regions with increased acetylated H3K27 (Fig. 2D; Table S2). Examples of genes with DOCs and DAc at the gene transcription start site (TSS) were CXCL10, IFI44L, APOBEC3A and MX1 (Fig. S3 and S4).

Covariates do not explain Mtb-triggered transcriptional and epigenetic differences among groups

The three groups differed with respect to several covariates (Table 1). Among the HC group, the proportion of females was higher, and the use of recreational drugs was lower compared to the PLWH and PrEP groups. Subjects in the PrEP group on average were younger compared to the two other groups. The duration of ART was longer for PLWH (mean $=12.12$ years, standard deviation $(\mathrm{SD})=7.64)$ than PrEP subjects $($ mean $=3$ years, $\mathrm{SD}=1,47)$. All PrEP subjects received Emtricitabine and Tenofovir disoproxil fumarate (TDF) while among PLWH there was a wider spread of ART drug regimens. However, only 4 out of 20 subjects had not received TDF at the time of enrolment (Table 1). Most of the inter-subject variability was accounted for by the analytical approach employed. Still, given the strong heterogeneity of study subjects we investigated the impact of smoking, recreational drug use and age on $M t b$-triggered chromatin 
and transcriptional responses. We found that recreational drug use did not explain the reduced transcriptional activity or absence of chromatin changes in response to $M t b$ among PLWH and PrEP subjects (Fig. 1F and Fig. 4A). Age and duration of ART/PrEP were also unlikely confounders since PLWH and PrEP subjects differed significantly in age and ART duration, yet both groups showed similar reduced transcriptional activity and lack of chromatin changes relative to $\mathrm{HC}$ subjects following challenge with $M t b$ (Fig. 4, B and C). Moreover, when stratifying $\mathrm{HC}$ and PLWH by age, HC subjects of 40 years and older showed pronounced $M t b$ triggered chromatin changes that were not observed for PLWH subjects (Fig. 4D). In addition to the covariates listed in Table 1, we also considered possible group-dependent differences in phagocytic activity. Since the yields of AM were generally not sufficient to conduct independent assessment of phagocytic activity, we used presence of Mtb reads in ATAC-seq libraries as proxy and failed to detect any significant group-dependent differences (Fig. 4E).

\section{Chromatin accessibility and binding of transcription factors}

To link Mtb-triggered epigenetic changes in AM from $\mathrm{HC}$ subjects with increased gene expression, we evaluated the enrichment of transcription factors (TFs) motifs in $M t b$-triggered DOC and DAc regions and found an enrichment of IFN-regulatory factor (IRF) motifs in those regions (Table 2). Next, we compared the average difference in TF footprint depth between $M t b$ challenged and non-challenge AM to estimate TF activity. Of the 682 TFs obtained from the JASPAR catalog, 21 had significantly higher activity (increased footprint depth) in DOC regions after $M t b$ challenge (Fig. 5A). Among these significant TFs were IRF9 (part of the antimycobacterial host response) and ZNF684 (a TB biomarker). Of note, $M t b$ challenge had triggered a higher fold change for IRF9 and ZNF684 RNA expression in AM from HC subjects 
compared to AM from PrEP or PLWH subjects (Fig. 5B). Moreover, IRF9 and ZNF684 footprints responsive to $M t b$ challenge were enriched in the transcription starting site (TSS) of genes that are part of the IFN signalling pathway $\left(p=8.9 \times 10^{-6}\right.$ and $p=0.03$, respectively) (Fig. 5C). Unexpectedly, we also observed an enrichment of IRF9 active footprints in the TSS of genes of the TNF signalling pathway $(p=0.01)$ (Fig. 5C). ZNF684 active footprints were observed in the core promoter of $T N F$ after $M t b$ challenge providing a direct link to a major proinflammatory cytokine (Fig. 5D). Moreover, in support of a coordinated effect of these two TFs, an active footprint for ZNF684 was observed in the promoter region of $I R F 9$ (Fig. 5D).

To further assess the link between $M t b$-dependent TF binding and RNA expression, we evaluated if the 135 expressed genes with active IRF9 footprint at the TSS were differentially expressed in HC following $M t b$ challenge. We observed that 65 out of the 135 genes were DEGs (57 upregulated and 8 down-regulated) following $M t b$ challenge $\left(p=7.9 \times 10^{-23}\right.$, Table S3 and Data File 5). AM from PLWH and PrEP had lower $\log F C$ in response to $M t b$ for all 65 DEGs (Data File 5). Of the 57 up-regulated DEGs with IRF9 TSS footprint, 17 were part of the IFN or TNF pathways, including NOD2 and ISG15 (Fig. 5D). Collectively, these data showed that the epigenetic response observed in AM from $\mathrm{HC}$ subjects promoted the binding of key transcriptions factors required for IFN and TNF signalling. The locked state of chromatin in AM from PLWH and PrEP subjects was therefore likely to account, at least in part, for the reduced transcriptional activity of those cells in response to $M t b$. 


\section{Discussion}

There is a scarcity of knowledge about the early fate of inhaled Mtb bacilli in the human lung. Infection of human hosts with $M t b$ is inferred from detectable anti-Mtb immunity through either the in-vivo tuberculin skin test (TST) or ex-vivo IFN- $\gamma$ release assays (IGRAs) (10). However, these tests reflect T-cell immunity that is being generated in the lymph nodes, and not in the lung, several weeks after the initial uptake of $M t b$ (11). Consequently, much of our knowledge about the $M t b$-host interplay from inhalation to the development of acquired $\mathrm{T}$ cell immunity has been derived from observations in animal models. In the mouse, $M t b$ bacilli in the lung alveoli are taken up by AM (7). Infected AM function as niche for Mtb replication prior to the migration of $M t b$-infected AM to the lung interstitium where $M t b$ are transferred to inflammatory macrophages that facilitate the induction of $M t b$-specific T cell immunity (7). The increased permissiveness of AM for $M t b$ in the mouse has been confirmed in a number of independent studies and appears to reflect the metabolic state of the cells $(12,13)$.

It is not known how well this scenario is replicated in the human lung. One critical difference between the mouse model and humans is the ability of a subgroup of $M t b$-exposed persons to avoid the development of classical T-cell immunity and TB disease even in the presence of long lasting $M t b$ exposure (9). Recently, the phenotype of strong resistance to immune conversion of persons living in high $M t b$ exposure settings has been confirmed for HIV-infected but immunereconstituted people (14). While the underlying mechanisms that preclude the development of classical T cell immunity are unknown, initial results implicate both non-classical T cells and B cells $(14,15)$. How and where AM interact with these cells of the acquired immunity arm is not known. We also do not know how the strong persistent exposure of human AM to environmental 
stimuli and pollutants impacts on AM physiology and anti-mycobacterial activity. An important influence of the environment on AM physiology is consistent with the pronounced interindividual differences observed in our study. Nevertheless, in our experiments all AM displayed an innate anti-mycobacterial immune response to $M t b$ that was dominated by IFN and TNF signalling suggesting that AM are directly involved in the host defense against $M t b$. However, this anti-Mtb response was significantly weaker in AM from PLWH and PrEP subjects.

The impetus for our experiments was the well-known observation that PLWH are at increased risk to become infected with $M t b$ and to develop clinical TB disease (4-6, 16). This increased susceptibility is still present in PLWH who have been immune-reconstituted with ART $(2,17)$. A detrimental effect of HIV on AM function has been shown in pre-ART patients including on their response to $M t b(18)$. In our study we found no evidence for reduced phagocytic uptake of Mtb by AM across phenotypic groups. This is consistent with previous reports that AM obtained from PLWH showed no defect in phagocytosis of Salmonella Typhimurium compared to HIVnegative controls (19). AM from PLWH on long term ART displayed a decreased ability to kill Streptococcus pneumoniae due to their HIV gp120-mediated reduced AM apoptotic activity (20, 21). Consistent with this finding, reduced AM phagocytic activity of Staphylococcus aureus in a sample of PLWH/ART subjects was associated with detectable proviral DNA (22). In our sample we found that 6 out of the 7 tested PLWH subjects had undetectable proviral counts arguing against a role of provirus in our sample (23). Moreover, the differences in the transcriptional response to $M t b$ between the PrEP and PLWH groups were less pronounced as compared to the differences of both groups to HC participants which argues against HIV being the main cause of the blunted anti-Mtb response by AM. 
The differences in the transcriptional response between the phenotype groups were graded and quantitative. However, the changes in $M t b$-triggered chromatin structure of AM were sharply divided between PLWH and PrEP subjects compared to controls. Indeed, AM from both PLWH receiving ART and PrEP subjects displayed a near total lack of chromatin changes in response to $M t b$. This suggested an unknown effect on chromatin of the drugs used in the PrEP and ART regimen. Yet, how drugs might impact the AM epigenetic responsiveness and the transcriptional response to $M t b$ is not clear. While the observed increased TF binding in DOC/DAc regions is consistent with the idea of the chromatin lock-down being the cause of the reduced transcriptional response, this causality remains to be more stringently established. Irrespective of the precise mechanisms, the blunted ability of AM to mount a response to an infectious insult may contribute to the known increased vulnerability to pulmonary disease of PLWH. For example, the prevalence of bacterial pneumonias and chronic obstructive pulmonary disease (COPD) are significantly increased in PLWH/ART patients $(24,25)$. PLWH/ART patients also experience an increased risk of sepsis with poor outcome (26). However, a more direct link of our findings can be made with TB for which PLWH on long term ART who have been immunereconstituted remain at increased risk $(2,17)$. Since our data suggest that the blunted AM response to $M t b$ is predominantly mediated by the anti-viral drugs and not the virus, use of PrEP in settings of high TB transmission might require to consider the risk of TB versus protection from HIV. To our knowledge, no data are presently available that link PrEP with risk of TB. We note, however, that PrEP has been associated with reduced risk of SARS-CoV-2 virus infection in Spain $(27,28)$. Efficient infection of epithelial cells by SARS-CoV-2 is dependent on the virus induced up-regulation of its ACE2 receptor (29). If the impact of PrEP on chromatin 
structure described here for AM also hold true for epithelial cells, this may underlie the protective effect observed.

\section{Materials and Methods}

\section{Subjects}

The study was approved by the Research Ethics Board of the McGill University Health Center (MUHC) (MP-CUSM-15-406). Participants were recruited at the McGill University Health Centre (MUHC, Montreal, Canada). HIV negative participants represented a control group $(n=23)$ and a high-risk group on preventive therapy (PrEP, $n=14)$. HIV positive participants (PLWH, $n=27)$ were receiving antiretroviral therapy (ART) to suppress peripheral viral load for at least 3 years and without any active respiratory symptoms or infections. Exclusion criteria included past history of TB, pregnancy, chronic cardiovascular or active pulmonary infection and immunosuppressive medication. All participants were tested with QuantiFERON Gold, Cat\#0594-2010/Cat\#T0590-0301 (Qiagen, Germany) and one subject each of the HC and PrEP groups tested positive. Since exclusion of these two subjects had no significant impact on reported results, we left them included in the present presentation of the data. Information on participant characteristics and antiretroviral drugs was extracted from the clinical database and participant electronic records. Of these enrolled subjects, we excluded 7 HC and 7 PLWH from the analyses due to low yield of BAL cells, or inability to pass quality control for sample or library preparation.

Bronchoalveolar lavage and sample processing 
Bronchoscopies were performed at the Centre for Innovative Medicine of the Research Institute of the MUHC following the guidelines of the American Thoracic Society (30). Briefly, standard flexible bronchoscopy of the middle lobe was conducted under local anesthesia with lidocaine with additional conscious sedation with intravenous midazolam. A total of $200 \mathrm{ml}$ sterile saline instilled in 50-ml volumes resulted in an average return of $115 \mathrm{ml}$ (range of 50-150 ml) of bronchoalveolar lavage (BAL) fluid. BAL fluid in non-adherent tubes was stored on ice for a maximum of 30 min prior to the isolation of AM. All BAL fluids were strained through a $100 \mu \mathrm{m}$ filter to remove any mucus and cell clumps. Cytology assessment of BAL samples indicated that AM represented $>90 \%$ of all recovered mononuclear cells.

\section{Alveolar macrophage preparation}

$\mathrm{AM}$ were isolated by adhesion. Collected BAL cells were spun for 10 minutes at $300 \mathrm{~g}, 10^{\circ} \mathrm{C}$, and washed twice in RPMI-1640 with L-glutamine (Wisent, Canada), containing 2\% human serum (heat-inactivated $\mathrm{AB}+$ off the clot, Wisent, Canada), 1\% penicillin/streptomycin (Gibco, USA), 10mM HEPES (Gibco, USA), 1\% Non-essential amino acids (Gibco, USA) and $1.75 \mathrm{ng} / \mathrm{ml}$ Amphotericin B (Wisent, Canada). After the cell count, 1.5x106 AM were seeded per well of a 6-well plate (Falcon, USA) in RPMI-1640 supplemented with 10\% human serum and incubated for $1.5 \mathrm{~h}-2 \mathrm{~h}$ at $37^{\circ} \mathrm{C}, 5 \% \mathrm{CO} 2$, and $95 \%$ relative humidity. Non-adherent cells were removed by thorough washings with pre-warmed $\left(37^{\circ} \mathrm{C}\right)$ RPMI-1640 supplemented with $2 \%$ human serum. Washed adherent AM were then infected with freshly prepared $M t b$. An estimate for the number of adherent AM was derived by subtracting the number of detached from the number of seeded cells. 
medRxiv preprint doi: https://doi.org/10.1101/2021.01.26.21250318; this version posted January 29, 2021. The copyright holder for this preprint (which was not certified by peer review) is the author/funder, who has granted medRxiv a license to display the preprint in perpetuity.

It is made available under a CC-BY-NC-ND 4.0 International license .

Virulent $M t b$ strain H37Rv was grown in a liquid culture of Middlebrook 7H9 medium (Difco, Detroit, USA, \#295939) containing 0.2\% glycerol (Fisher), 0.05\% Tween-80 (Sigma-Aldrich) and $10 \%$ albumin-dextrose-catalase $\left(\mathrm{ACD}\right.$, Fisher) at $37^{\circ} \mathrm{C}$ in rolling incubators. Bacteria were grown to log phase, as determined by an optical density of 0.3 to 0.8 at $600 \mathrm{~nm}$, prior to inoculum preparation. Further, bacterial cultures were spun for 15 minutes at $3700 \mathrm{rpm}$, resuspended in RPMI-1640 and dislodged with a $22 \mathrm{G}$ needle. Cell suspensions were filtered through $5 \mu \mathrm{m}$ filters (Millipore, USA) to ensure single mycobacteria suspensions for the challenge experiments. Bacterial counts of inocula were done using disposable Neubauer hemocytometer (C-Chip, INCYTO, South Korea). Bacterial loads were confirmed by colony-forming unit (CFU) counts by plating serial dilution of inoculum in 7H9 growth medium on Middlebrook 7H10 agar (Becton Dickinson, Franklin Lakes, NJ, USA, \#254520) plates containing 0.5\% glycerol (Fisher) and 10\% oleic acid-albumin-dextrose-catalase (OADC, Becton Dickinson, Franklin Lakes, USA, \#211886). Colonies were counted 3-4 weeks post-plating. AM were infected at a multiplicity of infection (MOI) of 5:1 for $18-20 \mathrm{hrs} 37^{\circ} \mathrm{C}, 5 \% \mathrm{CO} 2$, and $95 \%$ relative humidity.

\section{Preparation of RNASeq and ATACSeq libraries}

For downstream RNA and ATAC experiments AM were rinsed with warm PBS to remove damaged cells and debris before further processing. For RNA, cells were lysed with Trizol and kept at $-80^{\circ} \mathrm{C}$ until extraction. For RNA isolation the miRNeasy kit (Qiagen, Germany) was used and RNA integrity (RIN) was assessed with the Agilent 2100 Bioanalyzer (Agilent Technologies, Waldbronn, Germany). Only samples with RIN $>8$ were selected for library preparation using TruSeq RNA Library Preparation Kit v2, Set A (Illumina, USA).

For chromatin accessibility using the ATACseq method (31, 32); AM were lifted with pre-warmed CellStripper solution (Corning, USA) and gentle pipetting. Viability was assessed with trypan 
blue staining, and only samples with viability $>85 \%$ were used. We followed the protocol by

Buenrostro et al. (32). Briefly, 50,000 cells were used for generating challenged and nonchallenged libraries. AM were permeabilized with lysis buffer containing 0.05\% IGEPAL. Nuclei were incubated with Tn5 transposase (Illumina, USA) and further ATAC-seq libraries were amplified for a total of 5-10 PCR cycles. Barcoded and multiplexed RNA or ATAC-seq libraries were sequenced on an Illumina HiSeq4000 sequencer at Genome Quebec, Montreal, Canada.

\section{ChIP-seq library preparation and sequencing.}

Samples from challenged and non-challenged AM were crosslinked with $1 \% \mathrm{w} / \mathrm{v}$ formaldehyde for $10 \mathrm{~min}$ at RT and immediately quenched for $5 \mathrm{~min}$ with $125 \mathrm{mM}$ Glycine at RT. The formaldehyde fixed samples were then sonicated to 150-500 bp using a S220 (Covaris) and then ChIP-DNA prepared using a manual chromatin immunoprecipitation method with an AntibodyAntigen incubation of 18 hrs., followed by bead incubation for 135 minutes, and $6 \times 5$-minutes washing steps.

Approximately 0.5 million cells were used for each ChIP and $~ 50,000$ cells for the input. We used H3K27ac antibody (Abcam, Ab4729, GR3211959-1). ChIP and Input libraries were prepared using the MicroPlex Library Preparation Kit (C05010012, Diagenode), with alterations including PCR enrichment ( 7 to 20 cycles) prior to size selection and use of Ampure beads for size selection (250-350 bp). Libraries for ChIPSeq were sequenced on an Illumina NovaSeq 6000 S2 SR100.

\section{Data processing and statistical analysis}

Detailed description for raw sequences processing to statistical analyses are provided in Supplementary Information. Briefly, Baseline and challenged transcriptome and epigenomic landscapes of AMs were determined following published protocols $(31,33)$. Gene expression 
readouts were generated using alignments and annotated set of transcripts as input to Salmon (34). Differences in chromatin accessibility and H3K27ac levels were assessed with a countbased peak quantification as shown in (35). Quantification files from each approach were merged as a single matrix, genes and peaks with low counts were filtered out and libraries were normalized, scaled and $\log 2$ transformed using edgeR and Limma-voom $(36,37)$.

For the statistical analysis, we defined linear models with blocking design on subject ID to account for inter-individual differences and modelled the challenge effect nested within phenotypic groups. For all experiments we tested the challenge effect for HC, PrEP and PLWH subjects, while for RNAseq we also contrasted the phenotype groups against each other (interaction test). ATACseq and ChIPseq p-values were adjusted with Storey's false discovery rate (FDR) and peaks considered significant if FDR $<0.05$ and absolute log fold-change $(\operatorname{logFC})$ $>0.2$. These were termed differentially open chromatin (DOC) for ATACseq and differentially acetylated (DAc) when referring to ChIPseq. For RNA-seq we employed a two-stage FDR procedure and we considered genes to be differentially expressed genes (DEG), if absolute $\log \mathrm{FC} \geq 0.2$ and stageR $p$-val $\leq 0.05(38)$

We performed pathway and gene ontology enrichment analysis using three independent databases (KEGG, Reactome and AmiGO). Enrichment results were merged and the BenjaminiHochberg FDR method was applied to raw p-values. We tested TF motif enrichment in DOCs and DAc regions using HOMER (39). Mtb-triggered differences in TF footprint activity over DOC regions were estimated with HINT-ATAC (40). TF motifs with FDR $<0.05$ and activity score $>0.005$ were considered significant. 
Acknowledgments: We thank all volunteers who participated in this study. We thank Pilar Domenech for help with $M t b$ cultures. We thank personnel of the CL3 platform of the RI-MUHC and the Centre d'expertise et de services Génome Québec. We thank all members of the Schurr and Barreiro labs for discussions of the data and helpful comments on the manuscript.

Funding: This work was supported by grant 1R01AI124349 from the National Institutes of Health, USA, to ES and LBB; and in part by the Fonds de la Recherche Québec-Santé (FRQ-S) Réseau SIDA/Maladies infectieuses and Thérapie cellulaire (JPR). JS is supported through grants PID2019-106859GA-I00 and RYC-2017-23560 from the Spanish Ministry of Science and Innovation (MICINN). This research was supported through a resource allocation in the Cedar high performance computing cluster by Compute Canada and WestGrid.

Author Contributions: W.C.-M. analysed the transcriptomic data and V.M.F. analysed the epigenomic data. E.S., L.B.B., W.C.-M. and V.M.F designed the study. M.O. and P.C. prepared samples, and performed cell-based experiments for RNA-seq, ATAC-seq and ChIP-seq. A.D., R.H.M.S. and V.Y. performed ChIP experiments. J.-P.R. and J.G enrolled subjects. R.O. performed bronchoalveolar lavages. J.S. and A.P. provided analytical guidance and participated in initial data analyses. B.K. and C.L. provided experimental guidance for AM isolation from BAL samples. E.S. and L.B.B. procured funding sources W.C.-M., V.M.F., L.B.B. and E.S. wrote the draft of the manuscript. All authors participated in the final assemble of the manuscript. E.S. conceptualized and supervised the project.

Competing Interests: The authors declare no competing interests.

Data and materials availability: All data associated with this study are present in the main text or the Supplementary Materials. RNA, ATAC and ChIP-seq raw sequences and corresponding 
medRxiv preprint doi: https://doi.org/10.1101/2021.01.26.21250318; this version posted January 29, 2021. The copyright holder for this preprint (which was not certified by peer review) is the author/funder, who has granted medRxiv a license to display the preprint in perpetuity.

It is made available under a CC-BY-NC-ND 4.0 International license .

quantification matrices analysed is this work were submitted to NCBI's Gene Expression

Omnibus repository (data is being processed, accession number will be available for

publication).

\section{References}

1. W. H. Organization, Global tuberculosis report 2019., (2019).

2. S. D. Lawn, R. J. Wilkinson, ART and prevention of HIV-associated tuberculosis. Lancet HIV 2, e221-222 (2015).

3. S. Narayanan, S. Swaminathan, P. Supply, S. Shanmugam, G. Narendran, L. Hari, R. Ramachandran, C. Locht, M. S. Jawahar, P. R. Narayanan, Impact of HIV infection on the recurrence of tuberculosis in South India. J Infect Dis 201, 691-703 (2010).

4. P. Sonnenberg, J. Murray, J. R. Glynn, S. Shearer, B. Kambashi, P. Godfrey-Faussett, HIV-1 and recurrence, relapse, and reinfection of tuberculosis after cure: a cohort study in South African mineworkers. Lancet 358, 1687-1693 (2001).

5. A. C. Crampin, J. N. Mwaungulu, F. D. Mwaungulu, D. T. Mwafulirwa, K. Munthali, S. Floyd, P. E. Fine, J. R. Glynn, Recurrent TB: relapse or reinfection? The effect of HIV in a general population cohort in Malawi. AIDS 24, 417-426 (2010).

6. R. M. Houben, A. C. Crampin, R. Ndhlovu, P. Sonnenberg, P. Godfrey-Faussett, W. H. Haas, G. Engelmann, C. J. Lombard, D. Wilkinson, J. Bruchfeld, S. Lockman, J. Tappero, J. R. Glynn, Human immunodeficiency virus associated tuberculosis more often due to recent infection than reactivation of latent infection. Int J Tuberc Lung Dis 15, 24-31 (2011).

7. S. B. Cohen, B. H. Gern, J. L. Delahaye, K. N. Adams, C. R. Plumlee, J. K. Winkler, D. R. Sherman, M. Y. Gerner, K. B. Urdahl, Alveolar Macrophages Provide an Early Mycobacterium tuberculosis Niche and Initiate Dissemination. Cell Host Microbe 24, 439-446 e434 (2018).

8. S. F. Ackley, R. S. Lee, L. Worden, E. Zwick, T. C. Porco, M. A. Behr, C. S. Pepperell, Multiple exposures, reinfection and risk of progression to active tuberculosis. $R$ Soc Open Sci 6, 180999 (2019).

9. J. D. Simmons, C. M. Stein, C. Seshadri, M. Campo, G. Alter, S. Fortune, E. Schurr, R. S. Wallis, G. Churchyard, H. Mayanja-Kizza, W. H. Boom, T. R. Hawn, Immunological mechanisms of human resistance to persistent Mycobacterium tuberculosis infection. Nat Rev Immunol 18, 575-589 (2018).

10. U. Mack, G. B. Migliori, M. Sester, H. L. Rieder, S. Ehlers, D. Goletti, A. Bossink, K. Magdorf, C. Holscher, B. Kampmann, S. M. Arend, A. Detjen, G. Bothamley, J. P. Zellweger, H. Milburn, R. Diel, P. Ravn, F. Cobelens, P. J. Cardona, B. Kan, I. Solovic, R. Duarte, D. M. Cirillo, C. Lange, Tbnet, LTBI: latent tuberculosis infection or lasting immune responses to M. tuberculosis? A TBNET consensus statement. Eur Respir J 33, 956-973 (2009).

11. A. J. Wolf, L. Desvignes, B. Linas, N. Banaiee, T. Tamura, K. Takatsu, J. D. Ernst, Initiation of the adaptive immune response to Mycobacterium tuberculosis depends on antigen production in the local lymph node, not the lungs. J Exp Med 205, 105-115 (2008).

12. L. Huang, E. V. Nazarova, S. Tan, Y. Liu, D. G. Russell, Growth of Mycobacterium tuberculosis in vivo segregates with host macrophage metabolism and ontogeny. J Exp Med 215, 1135-1152 (2018).

13. A. C. Rothchild, G. S. Olson, J. Nemeth, L. M. Amon, D. Mai, E. S. Gold, A. H. Diercks, A. Aderem, Alveolar macrophages generate a noncanonical NRF2-driven transcriptional response to Mycobacterium tuberculosis in vivo. Sci Immunol 4, (2019).

14. E. E. Kroon, C. J. Kinnear, M. Orlova, S. Fischinger, S. Shin, S. Boolay, A. Jacobs, R. J. Wilkinson, G. Alter, E. Schurr, E. G. Hoal, M. Möller, Mycobacterium tuberculosis infection resistance in HIV-infected individuals,. Ebiomedicine, (2020). 
medRxiv preprint doi: https://doi.org/10.1101/2021.01.26.21250318; this version posted January 29, 2021. The copyright holder for this preprint (which was not certified by peer review) is the author/funder, who has granted medRxiv a license to display the preprint in perpetuity. It is made available under a CC-BY-NC-ND 4.0 International license .

15. L. L. Lu, M. T. Smith, K. K. Q. Yu, C. Luedemann, T. J. Suscovich, P. S. Grace, A. Cain, W. H. Yu, T. R. McKitrick, D. Lauffenburger, R. D. Cummings, H. Mayanja-Kizza, T. R. Hawn, W. H. Boom, C. M. Stein, S. M. Fortune, C. Seshadri, G. Alter, IFN-gamma-independent immune markers of Mycobacterium tuberculosis exposure. Nat Med 25, 977-987 (2019).

16. P. Sonnenberg, J. R. Glynn, K. Fielding, J. Murray, P. Godfrey-Faussett, S. Shearer, How soon after infection with HIV does the risk of tuberculosis start to increase? A retrospective cohort study in South African gold miners. J Infect Dis 191, 150-158 (2005).

17. A. Gupta, R. Wood, R. Kaplan, L. G. Bekker, S. D. Lawn, Tuberculosis incidence rates during 8 years of followup of an antiretroviral treatment cohort in South Africa: comparison with rates in the community. PLoS One 7, e34156 (2012).

18. K. C. Jambo, D. H. Banda, A. M. Kankwatira, N. Sukumar, T. J. Allain, R. S. Heyderman, D. G. Russell, H. C. Mwandumba, Small alveolar macrophages are infected preferentially by HIV and exhibit impaired phagocytic function. Mucosal Immunol 7, 1116-1126 (2014).

19. M. A. Gordon, S. B. Gordon, L. Musaya, E. E. Zijlstra, M. E. Molyneux, R. C. Read, Primary macrophages from HIV-infected adults show dysregulated cytokine responses to Salmonella, but normal internalization and killing. AIDS 21, 2399-2408 (2007).

20. S. M. Yeligar, J. M. Ward, F. L. Harris, L. A. S. Brown, D. M. Guidot, S. K. Cribbs, Dysregulation of Alveolar Macrophage PPARgamma, NADPH Oxidases, and TGFbeta1 in Otherwise Healthy HIV-Infected Individuals. AIDS Res Hum Retroviruses 33, 1018-1026 (2017).

21. P. J. Collini, M. A. Bewley, M. Mohasin, H. M. Marriott, R. F. Miller, A. M. Geretti, A. Beloukas, A. Papadimitropoulos, R. C. Read, M. Noursadeghi, D. H. Dockrell, HIV gp120 in the Lungs of Antiretroviral Therapy-treated Individuals Impairs Alveolar Macrophage Responses to Pneumococci. Am J Respir Crit Care Med 197, 1604-1615 (2018).

22. S. K. Cribbs, J. Lennox, A. M. Caliendo, L. A. Brown, D. M. Guidot, Healthy HIV-1-infected individuals on highly active antiretroviral therapy harbor HIV-1 in their alveolar macrophages. AIDS Res Hum Retroviruses 31, 64-70 (2015).

23. C. T. Costiniuk, S. Salahuddin, O. Farnos, R. Olivenstein, A. Pagliuzza, M. Orlova, E. Schurr, C. De Castro, J. Bourbeau, J. P. Routy, P. Ancuta, N. Chomont, M. A. Jenabian, HIV persistence in mucosal CD4+ T cells within the lungs of adults receiving long-term suppressive antiretroviral therapy. AIDS 32, 2279-2289 (2018).

24. K. Crothers, L. Huang, J. L. Goulet, M. B. Goetz, S. T. Brown, M. C. Rodriguez-Barradas, K. K. Oursler, D. Rimland, C. L. Gibert, A. A. Butt, A. C. Justice, HIV infection and risk for incident pulmonary diseases in the combination antiretroviral therapy era. Am J Respir Crit Care Med 183, 388-395 (2011).

25. M. R. Gingo, G. K. Balasubramani, T. B. Rice, L. Kingsley, E. C. Kleerup, R. Detels, E. C. Seaberg, R. M. Greenblatt, S. Holman, L. Huang, S. H. Sutton, M. Bertolet, A. Morris, Pulmonary symptoms and diagnoses are associated with HIV in the MACS and WIHS cohorts. BMC Pulm Med 14, 75 (2014).

26. S. K. Cribbs, C. Tse, J. Andrews, N. Shenvi, G. S. Martin, Characteristics and Outcomes of HIV-Infected Patients With Severe Sepsis: Continued Risk in the Post-Highly Active Antiretroviral Therapy Era. Crit Care Med 43, 1638-1645 (2015).

27. O. Ayerdi, T. Puerta, P. Clavo, M. Vera, J. Ballesteros, M. E. Fuentes, V. Estrada, C. Rodríguez, J. Del Romero, Preventive Efficacy of Tenofovir/Emtricitabine Against Severe Acute Respiratory Syndrome Coronavirus 2 Among Pre-Exposure Prophylaxis Users. Open Forum Infect Dis 7, ofaa455 (2020).

28. J. Del Amo, R. Polo, S. Moreno, A. Díaz, E. Martínez, J. R. Arribas, I. Jarrín, M. A. Hernán, Incidence and Severity of COVID-19 in HIV-Positive Persons Receiving Antiretroviral Therapy : A Cohort Study. Ann Intern Med 173, 536-541 (2020).

29. M. Hoffmann, H. Kleine-Weber, S. Schroeder, N. Krüger, T. Herrler, S. Erichsen, T. S. Schiergens, G. Herrler, N. H. Wu, A. Nitsche, M. A. Müller, C. Drosten, S. Pöhlmann, SARS-CoV-2 Cell Entry Depends on ACE2 and TMPRSS2 and Is Blocked by a Clinically Proven Protease Inhibitor. Cell 181, 271-280.e278 (2020).

30. K. C. Meyer, G. Raghu, R. P. Baughman, K. K. Brown, U. Costabel, R. M. du Bois, M. Drent, P. L. Haslam, D. S. Kim, S. Nagai, P. Rottoli, C. Saltini, M. Selman, C. Strange, B. Wood, B. A. L. i. I. L. D. American Thoracic Society Committee on, An official American Thoracic Society clinical practice guideline: the clinical utility of bronchoalveolar lavage cellular analysis in interstitial lung disease. Am J Respir Crit Care Med 185, 10041014 (2012). 
medRxiv preprint doi: https://doi.org/10.1101/2021.01.26.21250318; this version posted January 29, 2021. The copyright holder for this preprint (which was not certified by peer review) is the author/funder, who has granted medRxiv a license to display the preprint in perpetuity. It is made available under a CC-BY-NC-ND 4.0 International license.

31. J. D. Buenrostro, P. G. Giresi, L. C. Zaba, H. Y. Chang, W. J. Greenleaf, Transposition of native chromatin for fast and sensitive epigenomic profiling of open chromatin, DNA-binding proteins and nucleosome position. Nat Methods 10, 1213-1218 (2013).

32. J. D. Buenrostro, B. Wu, H. Y. Chang, W. J. Greenleaf, ATAC-seq: A Method for Assaying Chromatin Accessibility Genome-Wide. Curr Protoc Mol Biol 109, 2129 21-21 2929 (2015).

33. A. Pacis, L. Tailleux, A. M. Morin, J. Lambourne, J. L. Maclsaac, V. Yotova, A. Dumaine, A. Danckaert, F. Luca, J. C. Grenier, K. D. Hansen, B. Gicquel, M. Yu, A. Pai, C. He, J. Tung, T. Pastinen, M. S. Kobor, R. Pique-Regi, Y. Gilad, L. B. Barreiro, Bacterial infection remodels the DNA methylation landscape of human dendritic cells. Genome Res 25, 1801-1811 (2015).

34. R. Patro, G. Duggal, M. I. Love, R. A. Irizarry, C. Kingsford, Salmon provides fast and bias-aware quantification of transcript expression. Nat Methods 14, 417-419 (2017).

35. M. R. Corces, J. M. Granja, S. Shams, B. H. Louie, J. A. Seoane, W. Zhou, T. C. Silva, C. Groeneveld, C. K. Wong, S. W. Cho, A. T. Satpathy, M. R. Mumbach, K. A. Hoadley, A. G. Robertson, N. C. Sheffield, I. Felau, M. A. A. Castro, B. P. Berman, L. M. Staudt, J. C. Zenklusen, P. W. Laird, C. Curtis, N. Cancer Genome Atlas Analysis, W. J. Greenleaf, H. Y. Chang, The chromatin accessibility landscape of primary human cancers. Science 362, (2018).

36. M. D. Robinson, D. J. McCarthy, G. K. Smyth, edgeR: a Bioconductor package for differential expression analysis of digital gene expression data. Bioinformatics 26, 139-140 (2010).

37. C. W. Law, Y. Chen, W. Shi, G. K. Smyth, voom: Precision weights unlock linear model analysis tools for RNAseq read counts. Genome Biol 15, R29 (2014).

38. K. Van den Berge, C. Soneson, M. D. Robinson, L. Clement, stageR: a general stage-wise method for controlling the gene-level false discovery rate in differential expression and differential transcript usage. Genome Biol 18, 151 (2017).

39. S. Heinz, C. Benner, N. Spann, E. Bertolino, Y. C. Lin, P. Laslo, J. X. Cheng, C. Murre, H. Singh, C. K. Glass, Simple combinations of lineage-determining transcription factors prime cis-regulatory elements required for macrophage and B cell identities. Mol Cell 38, 576-589 (2010).

40. Z. Li, M. H. Schulz, T. Look, M. Begemann, M. Zenke, I. G. Costa, Identification of transcription factor binding sites using ATAC-seq. Genome Biol 20, 45 (2019). 
medRxiv preprint doi: https://doi.org/10.1101/2021.01.26.21250318; this version posted January 29, 2021. The copyright holder for this preprint (which was not certified by peer review) is the author/funder, who has granted medRxiv a license to display the preprint in perpetuity.

\section{It is made available under a CC-BY-NC-ND 4.0 International license .}

\section{Figures}

A

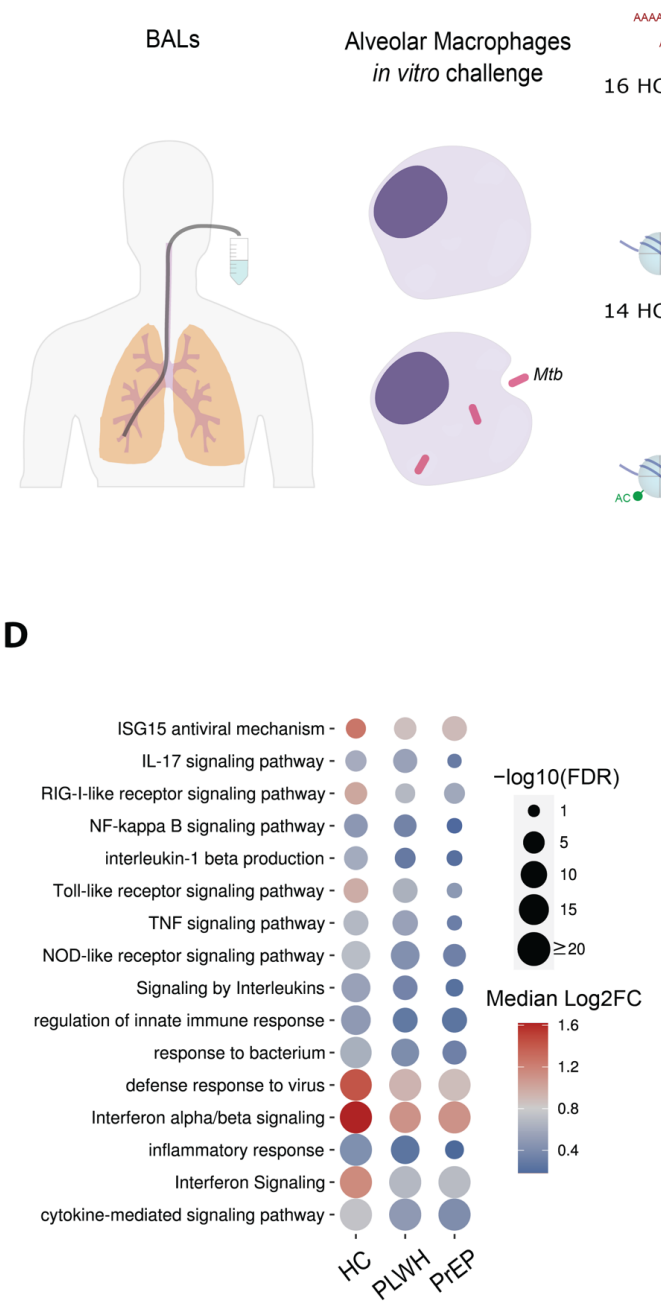

RNA-seq

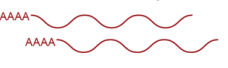

6 HC 20 PLWH 14 PrEP

ATAC-seq

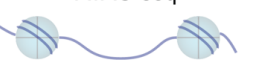

14 HC 17 PLWH 10 PrEP

ChIP-seq

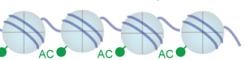

$4 \mathrm{HC} \quad 11 \mathrm{PLWH}$

$\mathbf{E}$

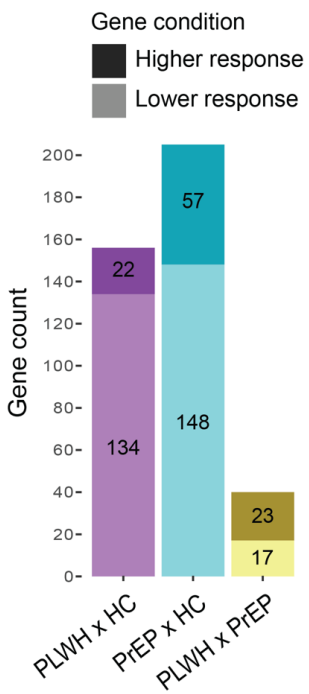

B

C

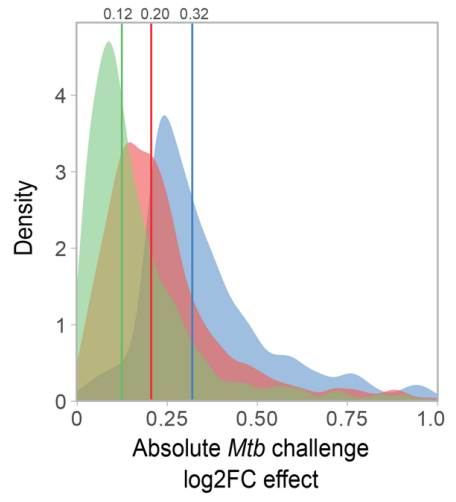

\section{$\mathbf{F}$}

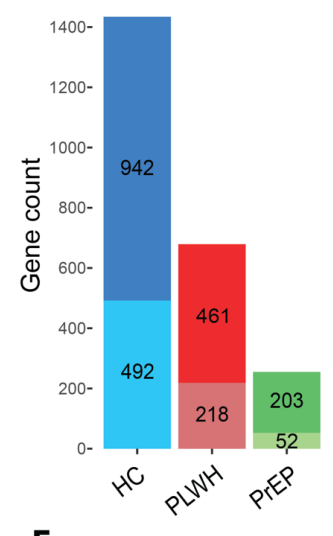

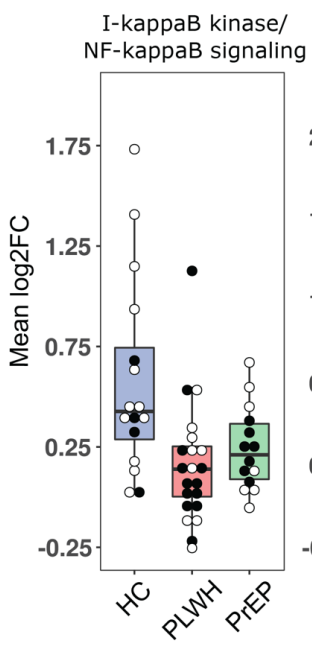
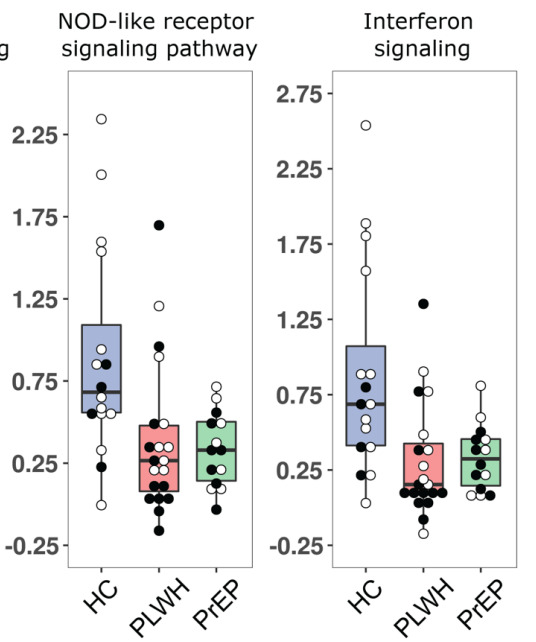

G

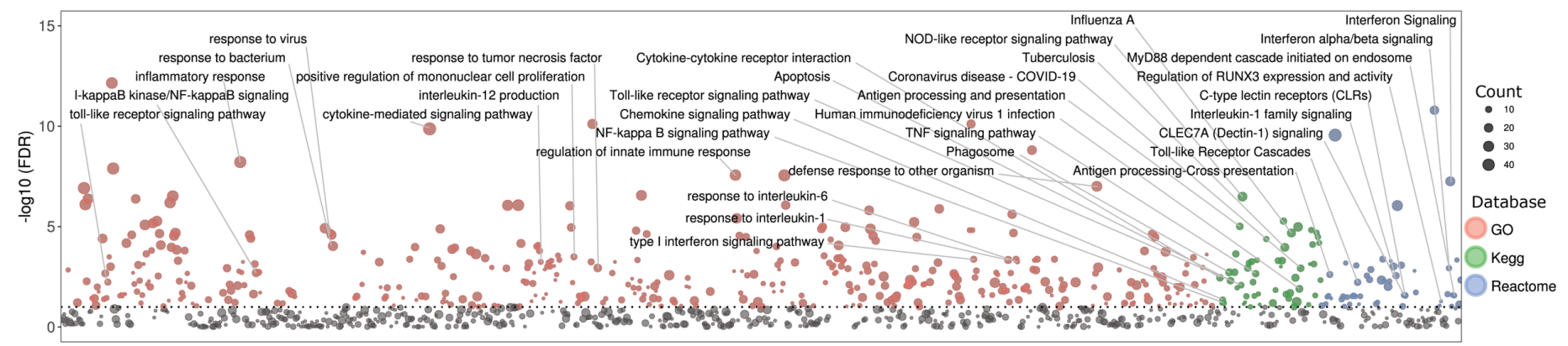

Fig. 1 Study design and results for differential gene expression by alveolar macrophages

(AM) after challenge with Mycobacterium tuberculosis (Mtb). 
(A) Experimental design and sample number by group. Each subject underwent bronchoalveolar lavage (BAL), AM were obtained from BAL and challenged in-vitro with $M t b$. RNA was obtained for RNA-seq, while DNA was used for ATAC-seq and ChIP-seq experiments. (B) Bar graph summarizing differentially expressed genes (DEG) by AM following $M t b$ challenge across the three phenotypic groups (healthy controls - HC; persons living with HIV on antiretroviral therapy - PLWH; subjects on pre-exposure prophylaxis with antiretroviral therapy - PrEP). The y-axis indicates the cumulative DEG count and group ID is indicated below each bar. Dark colour shades represent up-regulated genes (positive log fold-change; logFC) while light shades depict down-regulated genes (negative $\operatorname{logFC}$ ). (C) Density plot presenting $\operatorname{logFC}$ as absolute values. All DEGs from panel $\mathbf{B}$ had their $\log 2 \mathrm{FC}$ converted to absolute values and plotted using density function. Vertical coloured lines indicate the median of absolute values per group, with exact values given on top. Blue shade represents HC, red indicates PLWH and PrEP were coloured in green. (D) Dot plot presenting significance and overall effect for selected pathways and GO-terms. Pathways/GO-terms are listed on the left, dot sizes reflect the negative log10 FDR values for the enrichment test (bigger dots have smaller $p$-values) and colours from red (higher value) to blue (lower value) indicate the median $\log 2 \mathrm{FC}$ across all detected genes in the corresponding pathway or GO term. To derive the average $\log 2 \mathrm{FC}$ for a pathway/GO term, for each group we identified the corresponding DEGs, pooled all gene IDs from the three groups, retrieved the $\log 2 \mathrm{FC}$ for each of these genes and established the median of $\log 2 \mathrm{FC}$ per group. (E) Bar graph summarizing genes that displayed significantly different log2FC between groups (differential $M t b$ response). The y-axis indicates the cumulative DEG count while the x-axis indicates the pair-wise group contrast. Dark colour shading represents genes with higher response (e.g. $M t b$ effect in PLWH vs HC subjects resulted in a positive log2FC difference) 
while light shading depicts genes with lower response (negative log2FC difference). The union of all gene IDs identified across the three contrasts $(n=401)$ results in 333 unique gene IDs. (F) Boxplot for three selected pathway/GO terms for genes with significant differential $M t b$ response between PLWH or PrEP against controls $(n=302)$. For each term, the $y$-axis displays the mean $\log 2 \mathrm{FC}$ and dots represent per-subject mean value for pooled DEG. Phenotypic groups are indicated below each box plot across the three terms. White dots represent mean $\log 2 \mathrm{FC}$ for subjects who do not smoke cigarettes or used cannabis, while black dots depict smokers and/or cannabis users. To derive the subject mean $\log 2 \mathrm{FC}$, we used pooled DEG from the first two columns in panel E which were significant for interaction contrasts PLWH vs HC and PrEP vs $\mathrm{HC}$, obtained subject-wise $\log 2 \mathrm{FC}$ for each DEG and averaged the log2FC from all genes per subject. Results showed that group differences are not driven by outliers and that smoking, or cannabis consumption do not explain group differences. (G) Manhattan plot for enrichment test of pathways and GO-terms from merged DEG detected in the differential Mtb responses of PLWH vs HC and PrEP vs HC. The y-axis indicates the negative log 10 false discovery rate (FDR) values while the tested terms are arranged along the x-axis. The horizontal dashed line represents the 10\% FDR cut-off for significant pathways/GO-terms. Dots are sized as a function of the DEG number in a term and colors represent the database from which the terms were obtained. Identified terms represent innate immune processes and intracellular defense mechanisms. 
A

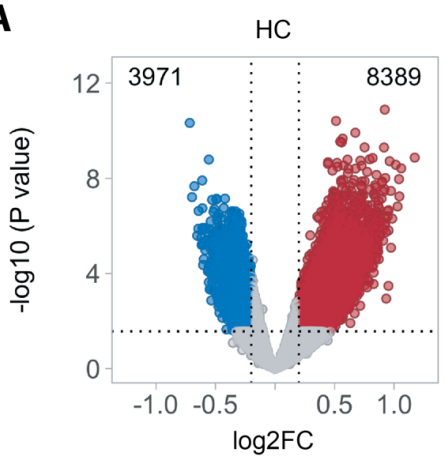

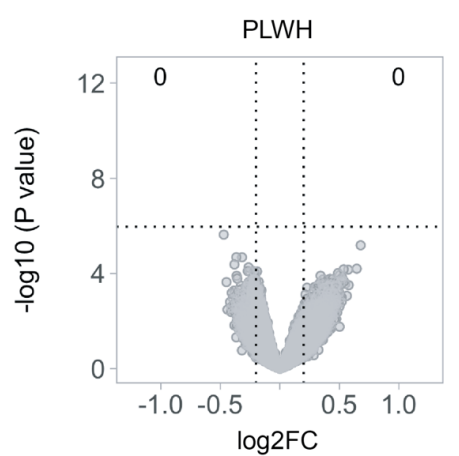

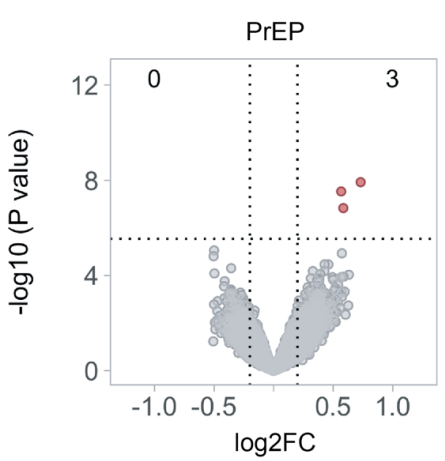

B

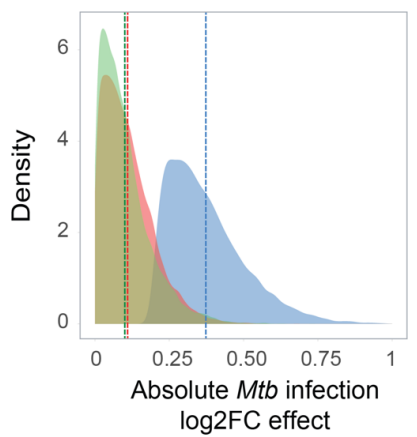

C

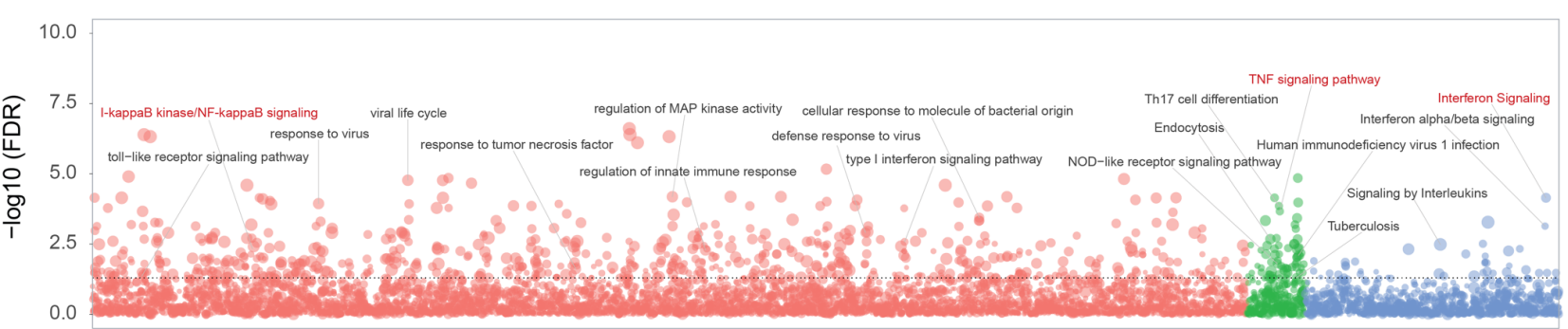

D

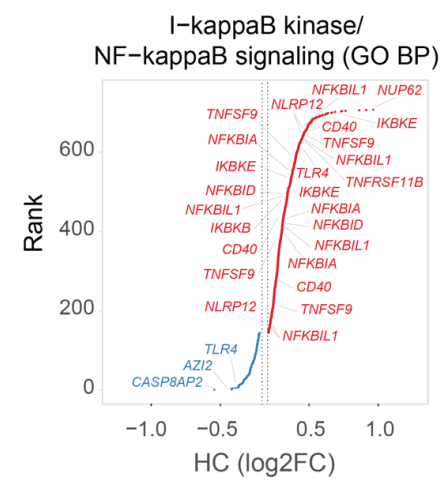

TNF signaling (KEGG)

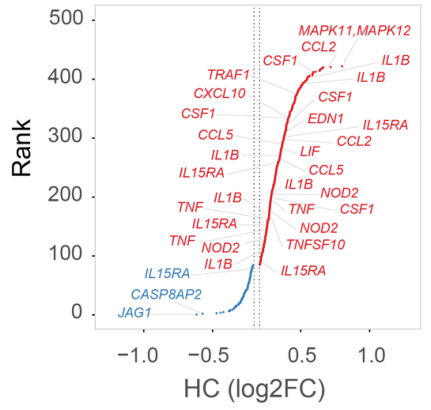

Interferon Signaling (Reactome)

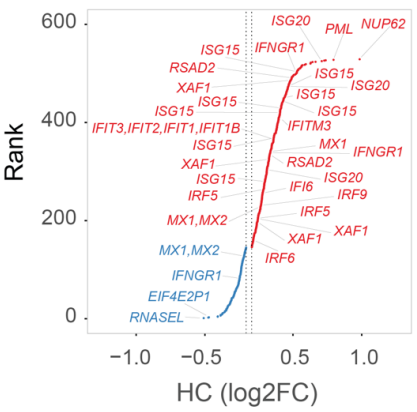

Fig. 2 Alveolar macrophage chromatin remodeling in response to $M t b$.

(A) Significance of chromatin changes in response to Mtb is plotted against the magnitude of the change. Significantly opened or closed chromatin regions at a false discovery rate (FDR) of 5\% after $M t b$ challenge are represented by dots marked in red or blue, respectively. The counts of differential open/closed chromatin (DOC) regions are given at the top left and right corners.

Dashed lines indicate the 5\% FDR on the y-axis and the minimum $\log 2 \mathrm{FC}$ of 0.2 on the $\mathrm{x}$-axis. In healthy controls (HC), $23.3 \%$ of the tested regions displayed significant chromatin 
remodelling in response to $M t b$, while subjects on pre-exposure prophylaxis (PrEP), and persons living with HIV receiving antiretroviral treatments (PLWH) lacked significant chromatin changes. (B) Density of the absolute log2FC effect for 12,360 DOC regions observed in the HC response to $M t b$ is shown for HC, PrEP, and PLWH subjects in blue, green, and red, respectively. The mean $\log 2 \mathrm{FC}$ for each group is highlighted by dashed horizontal lines. Both PrEP and PLWH groups show strongly impaired chromatin remodelling in response to Mtb compared to HC. (C) Pathway/GO-term enrichment analysis of genes assigned to DOC regions for the $\mathrm{HC}$ response to $M t b$. GO biological process (BP), KEGG, and Reactome pathways to which at least five genes had been assigned are plotted against the negative log 10 FDR. In total, 724 out of the 8066 tested terms are significant. Enrichment analysis indicated that in AM of HC subjects, $M t b$ challenge promoted chromatin remodelling in regions assigned to genes belonging to IFN and TNF signalling pathways as well as response to viral and bacterial pathogens. (D) Rank plot of selected GO BP, KEGG, and Reactome pathways. DOC regions in AM of HC subjects tagged genes assigned to "I-kappaB kinase/NF-kappaB signaling", "TNF signalling", and "Interferon signalling" pathways. DOC are plotted according to their $\log 2 \mathrm{FC}$ on the $\mathrm{x}$-axis and ranked according to the magnitude of change on the y-axis. Genes corresponding to opening or closing DOC regions are shown in red and blue, respectively. Horizontal doted lines indicate the $\log 2 \mathrm{FC}$ cut off of -0.2 and 0.2 . In the selected pathways, the majority of the chromatin changes observed indicated increased accessibility in response to $M t b$. 
A

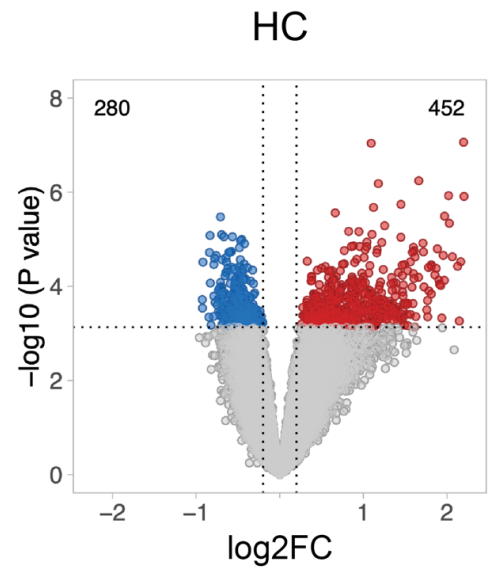

PLWH

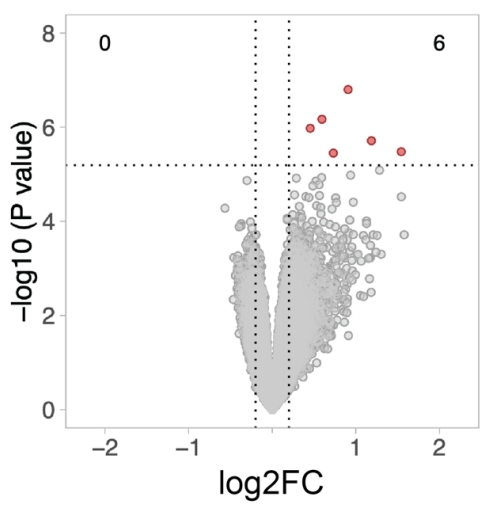

B

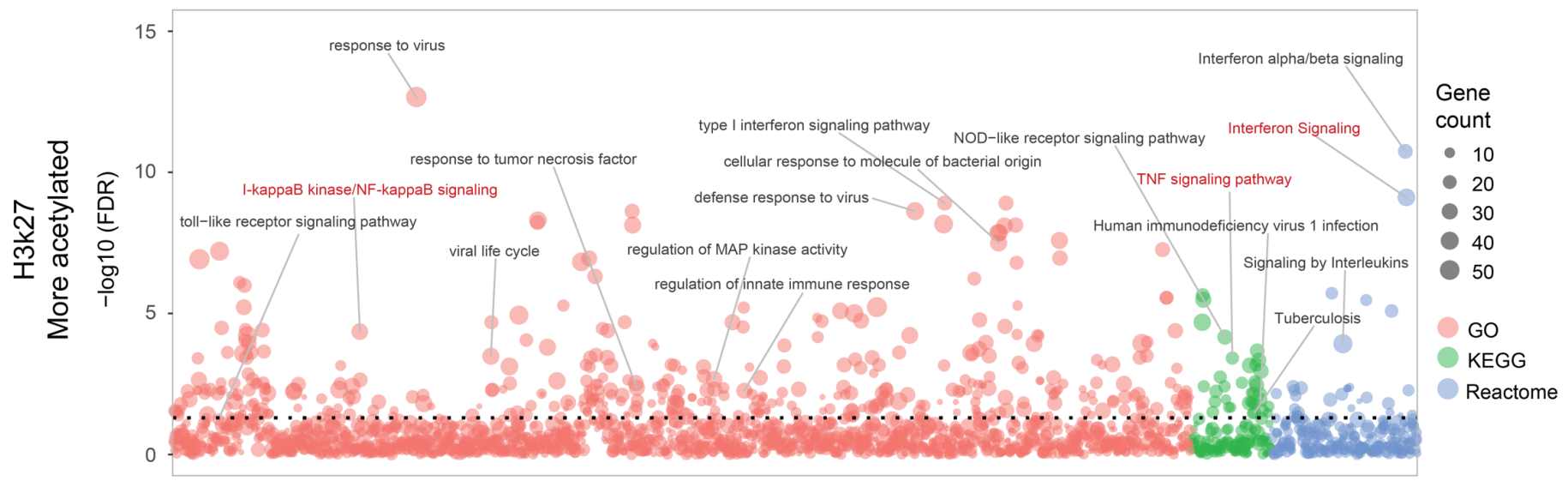

Fig. 3 Histone H3K27 acetylation changes in response to Mtb.

a, Volcano plots for $\mathrm{H} 3 \mathrm{~K} 27$ acetylation response to Mtb. Chromatin regions significantly acetylated at a false discovery rate (FDR) $<5 \%$ after $M t b$ challenge are marked in red and blue, respectively. The numbers of acetylated regions are given at the top. Significant changes in H3K27 acetylation, indicating active enhancers, were observed mostly for the healthy control (HC) group, which encompassed five subjects compared to 11 persons living with HIV on antiretroviral therapy (PLWH). While many peaks for H3K27ac did not pass FDR 5\%, the magnitude of $\log 2 \mathrm{FC}$ was higher in $\mathrm{HC}$ compared to PLWH. 
b, Enrichment analysis of genes assigned to regions with increased H3K27 acetylation for the $\mathrm{HC}$ response to $M t b$. GO biological processes (BP), KEGG, and Reactome pathways with at least five assigned genes are plotted against the negative $\log 10$ FDR. A total of 167 out of 906 tested terms were significant. As observed for the chromatin changes, enrichment analysis indicated that in $\mathrm{AM}$ of $\mathrm{HC}$ subjects, $M t b$ challenge promoted higher acetylation in regions assigned to genes belonging to IFN and TNF signalling pathways as well as response to viral and bacterial pathogens. 
medRxiv preprint doi: https://doi.org/10.1101/2021.01.26.21250318; this version posted January 29, 2021. The copyright holder for this preprint (which was not certified by peer review) is the author/funder, who has granted medRxiv a license to display the preprint in perpetuity.

It is made available under a CC-BY-NC-ND 4.0 International license.

A

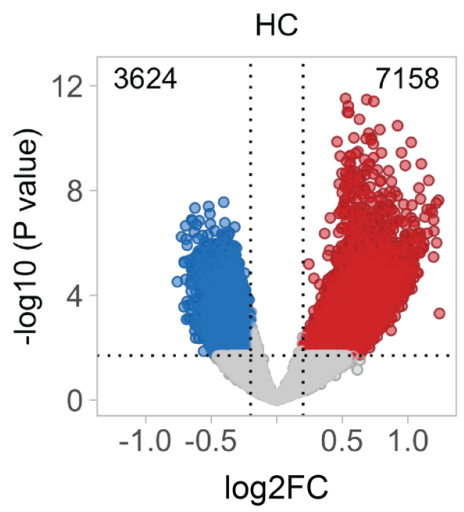

PLWH

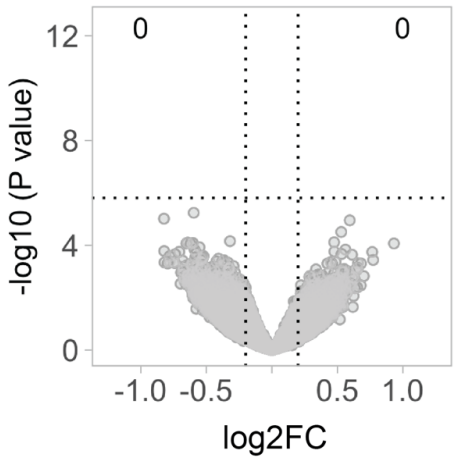

B

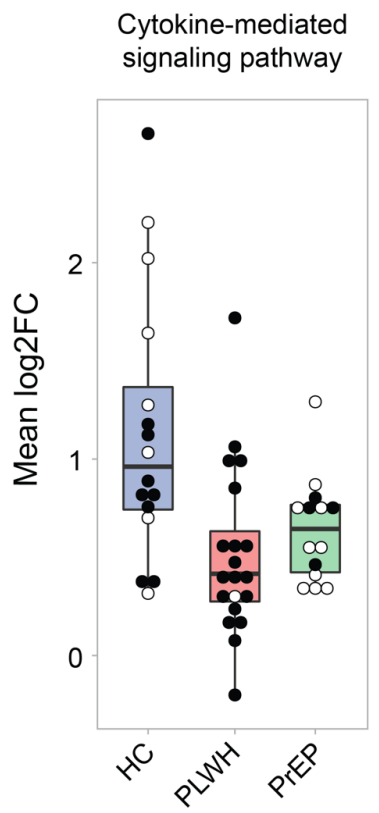

Interferon alpha/beta signaling

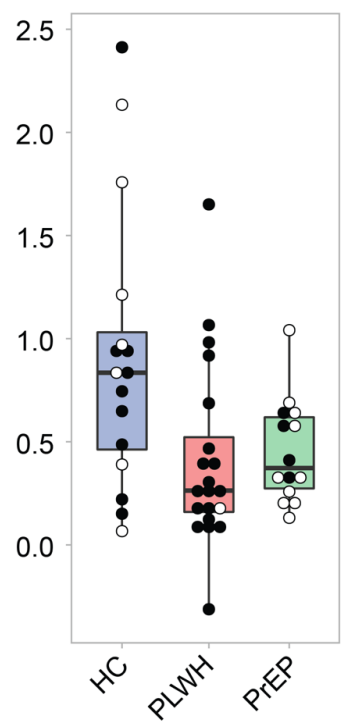

Defense response to virus

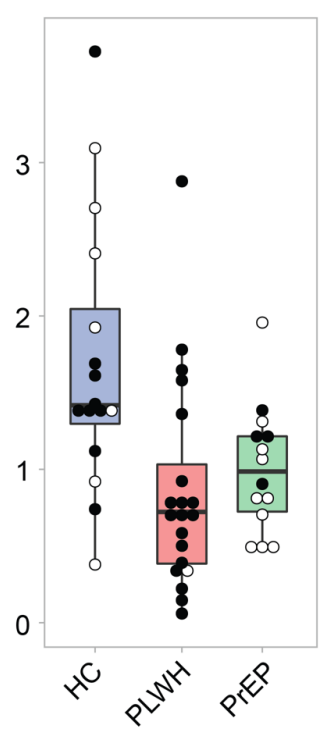

C

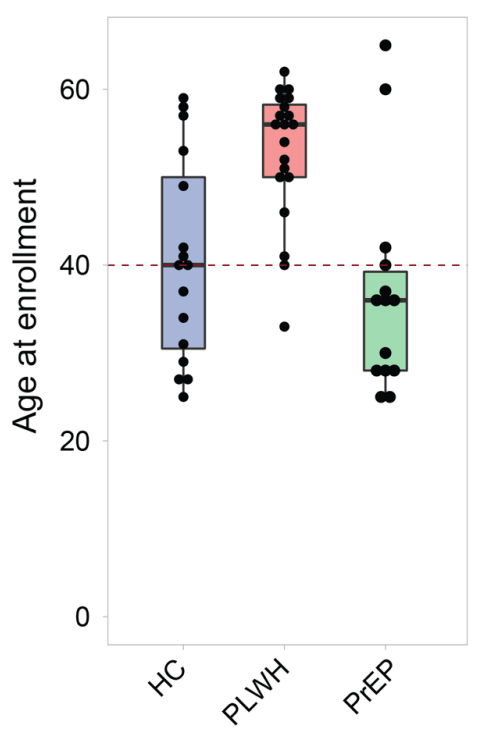

E

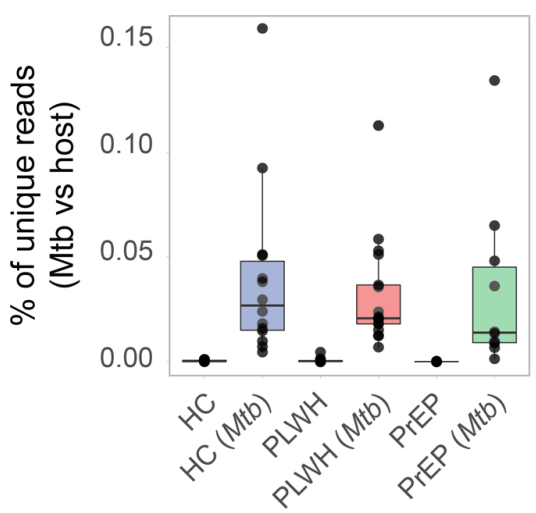




\section{Fig. 4. Impact of covariates on Mtb-triggered transcriptional and epigenetic changes in}

AM.

(A) Volcano plots for chromatin accessibility changes by AM in response to $M t b$ after removing subjects who smoked cigarettes, cannabis, or both. Included in this analysis were $10 \mathrm{HC}, 9$ PLWH, and 6 PrEP. Significantly opened or closed chromatin regions at a false discovery rate (FDR) of 5\% after $M t b$ challenge are represented by dots marked in red or blue, respectively. The counts of differential open/closed chromatin (DOC) regions are given at the top left and right corners. Dashed lines indicate the 5\% FDR on the y-axis and the minimum log2FC of 0.2 on the x-axis. (B) Boxplot for three selected pathway/GO terms for genes with significant differential $M t b$ response between PLWH or PrEP against HC $(n=302)$. For each term, the y-axis displays the mean $\log 2 \mathrm{FC}$ and dots represent per-subject mean value for pooled DEG from the three groups. White dots represent mean $\log 2 \mathrm{FC}$ for subjects younger than 40 years at enrolment, while black dots depict subjects $\geq 40$ years of age. To derive the subject mean log2FC, we used DEG from Fig. 1e which were part of significant pathway/GO terms, pooled the genes for each group, obtained the $\log 2 \mathrm{FC}$ per subject for each DEG and averaged the $\log 2 \mathrm{FC}$ from all genes per subject. (C) Boxplot showing the distribution of age at enrolment across the three studied groups. Subjects of the PLWH group were significantly older than the subjects of the healthy controls and PrEP groups. (D) Volcano plots for chromatin accessibility changes by AM in response to $M t b$ stratified by age for subjects of the HC and PLWH groups. Subjects tested were $\geq 40$ years at enrolment. In this age class the HC, PLWH and PrEP groups encompassed 9, 15 and 3 subjects, respectively. Due to low subject count PrEP subjects were excluded from this stratified analysis. Significantly opened or closed chromatin regions at a false discovery rate (FDR) of 5\% after $M t b$ challenge are represented by dots marked in red or blue, respectively. 
The counts of differential open/closed chromatin (DOC) regions are given at the top left and right corners. Dashed lines indicate the 5\% FDR on the $\mathrm{y}$-axis and the minimum $\log 2 \mathrm{FC}$ of 0.2 on the X-axis. (E) Boxplot of distribution for $M t b$-aligned reads in ATAC-seq experiments. The $\mathrm{y}$-axis indicates the proportion of total unique paired-end reads aligned to the $M t b \mathrm{H} 37 \mathrm{rv}$ genome relative to the Human genome hg38. Unique alignments to $M t b$ genome quantify DNA traces of phagocyted/lysed mycobacteria. In the absence of $M t b$ challenge, little to no alignment was observed to $M t b$ genome. In the challenge condition, the three groups (HC, PLWH, and PrEP) presented a range of proportions of $M t b$ unique reads. No statistical significance was observed between the proportion of $M t b$ reads between the phenotype groups. 
A

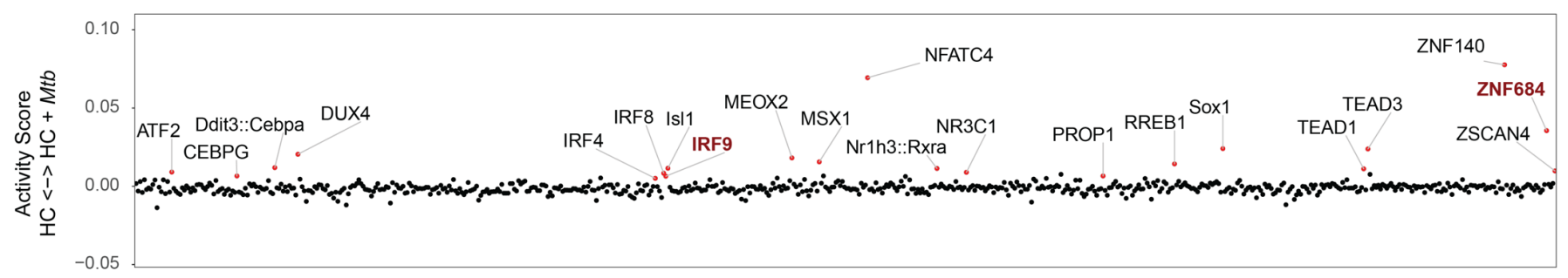

B

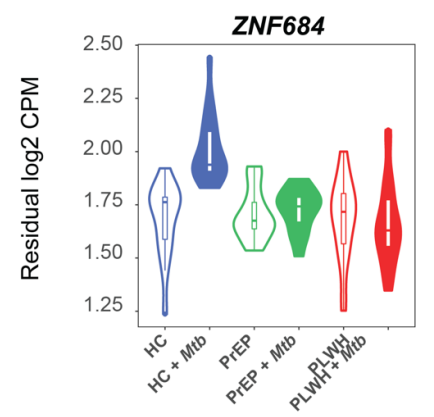

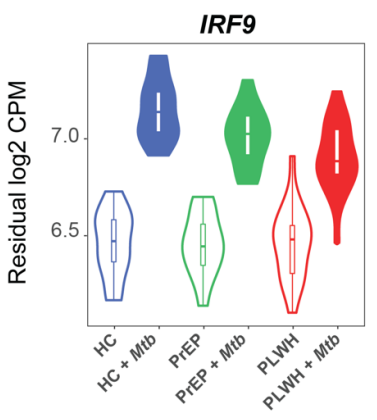

C

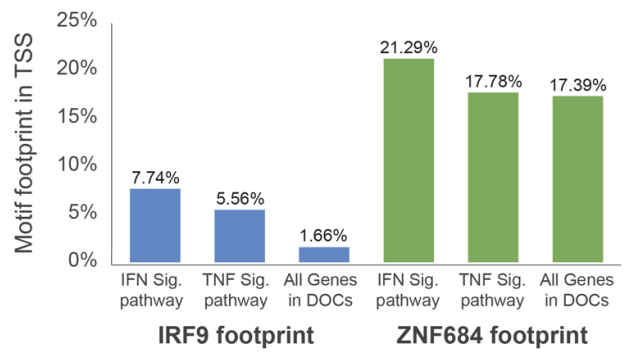

D

Chr6: $31,575,000-31,576,600$

Chr14: $24,160,500-24,162,000$
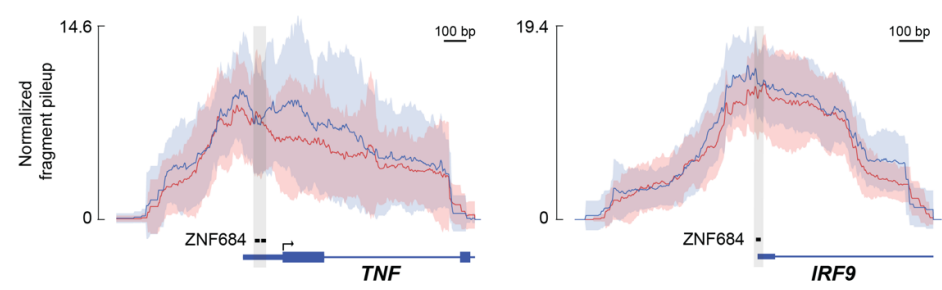

Chr16: 50,697,500-50,700,000

Chr1: 1,012,500-1,015,000
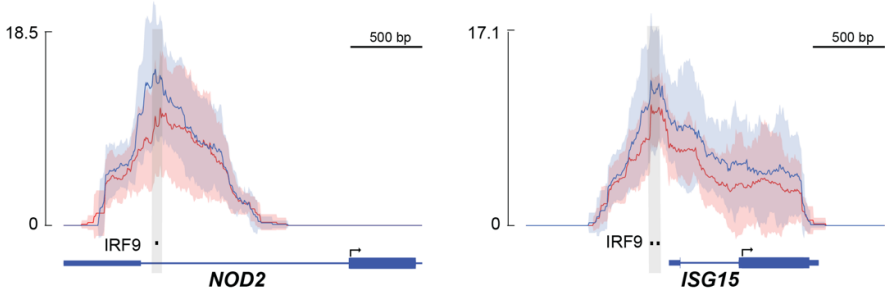

Fig. 5 Transcription factor (TF) footprint activity in differential open/closed chromatin (DOC) regions.

(A) Activity scores denoting differences in depth of TF footprints between healthy controls (HC) and $\mathrm{HC}+M t b \mathrm{AM}$ are plotted on the $\mathrm{y}$-axis for $682 \mathrm{TFs}$ from the JASPAR catalog showing a minimum of 50 footprints in DOC regions. A positive activity score indicates that footprints for a TF are more pronounced in $\mathrm{HC}$ after $M t b$ infection. Twenty-one TF with significant activity score at FDR $<5 \%$ are displayed as red dots. (B) mRNA expression levels for the transcription factors IRF9 and ZNF684 are shown as violin plots. The residual log2 copy per million (CPM) reads, after removing inter-individual variability, is plotted for each group i.e., healthy controls (HC), subjects on pre-exposure prophylaxis (PrEP), and persons living with HIV receiving 
antiretroviral treatments (PLWH) and condition (Mtb-challenged or not). (C) Percentage of genes in the TNF or IFN pathways that have IRF9 or ZNF684 footprints in their transcription starting site (TSS; $\pm 5 \mathrm{~kb}$ from the promoter) was compared to all genes with TSS in DOC regions. As expected, we observed that IRF9 footprints are more frequent in genes assigned to Reactome's IFN signalling pathway compared to random TSS in DOCs. Surprisingly, IRF9 footprints were also more frequent than by chance in KEGG's TNF pathway. (D) ZNF684 and IRF9 footprints in selected IFN and TNF genes. The mean normalized fragment pileup is plotted on the y-axis as red and blue lines for non-infected and Mtb-challenged AM from HC, respectively. The standard deviation of the means is shown by shades for each condition. The location of the motif assigned to the footprint is shown by a black bar at the bottom. We detected a pronounced footprint for ZNF684 in the core promoter of the $T N F$ gene and also in the promoter of IRF9. Motifs for IRF9 were detected in footprints located close to the promoter of the NOD2 and ISG15 genes. 
Table 1. Demographics and characteristics of enrolled subjects.

\begin{tabular}{|c|c|c|c|c|c|c|c|c|c|}
\hline SampleID & Sex & Age at recrutment & Smoker & Recreational drug & Medical history & ART & Prescription drug molecules & Sexually Transmitted Infections & CD4 count \\
\hline AMC2 & $\mathrm{M}$ & 240 & YES & Cannabis & None & NO & None & None & 495 \\
\hline AMC7 & M & $\geq 40$ & No & NO & HTN, T1DM, gout & NO & None & None & 500 \\
\hline AMC8 & M & $<40$ & No & NO & None & NO & None & None & 1536 \\
\hline AMC9 & M & $\geq 40$ & YES & Cannabis & None & No & None & None & 1290 \\
\hline AMC10 & M & $<40$ & NO & No & None & NO & None & None & 1091 \\
\hline AMC11 & M & $<40$ & No & No & None & No & None & None & 439 \\
\hline AMC12 & M & $\geq 40$ & NO & NO & None & NO & None & None & 1263 \\
\hline AMC13 & $\mathrm{F}$ & $<40$ & No & No & None & NO & None & None & 871 \\
\hline AMC14 & $\mathrm{F}$ & $\geq 40$ & YES & NO & None & NO & None & None & 1023 \\
\hline AMC15 & $\mathrm{F}$ & $\geq 40$ & NO & NO & None & NO & None & None & 843 \\
\hline AMC16 & $\mathrm{F}$ & $<40$ & No & No & None & No & None & None & 1249 \\
\hline AMC18 & M & $<40$ & NO & NO & None & NO & None & None & 826 \\
\hline AMC19 & M & $\geq 40$ & NO & NO & None & NO & None & None & 692 \\
\hline AMC20 & M & $<40$ & NO & NO & None & NO & None & None & 545 \\
\hline AMC21 & M & $\geq 40$ & No & NO & HTN, T2DM & NO & None & None & 1077 \\
\hline AMC22 & $\mathrm{F}$ & $\geq 40$ & YES & Cannabis & None & NO & None & None & 1248 \\
\hline AMP23 & M & $\geq 40$ & YES & Cannabis & fibromyalgia & PrEP (2017) & Emtricitabine, Tenofovir_DF & None & 819 \\
\hline AMP25 & M & $<40$ & NO & NO & ADHD, depression & $\operatorname{PrEP}(2016)$ & Emtricitabine, Tenofovir_DF & None & 575 \\
\hline AMP26 & M & $<40$ & NO & Cannabis & chronic.alcoholism & $\operatorname{PrEP}(2015)$ & Emtricitabine, Tenofovir_DF & Syphilis & 835 \\
\hline AMP27 & M & $<40$ & No & NO & None & PrEP (2014) & Emtricitabine, Tenofovir_DF & Chlamydia and Gonorrhea & 961 \\
\hline AMP28 & M & $\geq 40$ & YES & No & HTN, T1DM, ADHD & PrEP (2016) & Emtricitabine, Tenofovir_DF & None & 1254 \\
\hline AMP29 & M & $<40$ & YES & Cannabis & None & $\operatorname{PrEP}(2013)$ & Emtricitabine, Tenofovir_DF & Chlamydia & 981 \\
\hline AMP30 & M & $<40$ & NO & No & depression & $\operatorname{PrEP}(2016)$ & Emtricitabine, Tenofovir_DF & None & 847 \\
\hline AMP31 & M & $<40$ & No & No & None & PrEP (2016) & Emtricitabine, Tenofovir_DF & None & 1163 \\
\hline AMP32 & M & $<40$ & NO & NO & None & PrEP (2017) & Emtricitabine, Tenofovir_DF & None & 1069 \\
\hline AMP33 & M & $<40$ & No & Speed & depression & PrEP (2019) & Emtricitabine, Tenofovir_DF & None & 691 \\
\hline AMP34 & M & $\geq 40$ & No & NO & None & $\operatorname{PrEP}(2016)$ & Emtricitabine, Tenofovir_DF & Chlamydia & 208 \\
\hline AMP35 & M & $<40$ & YES & Cocaine & None & $\operatorname{PrEP}(2018)$ & Emtricitabine, Tenofovir_DF & Chlamydia & 832 \\
\hline AMP36 & M & $<40$ & YES & Ketamine & None & $\operatorname{PrEP}(2016)$ & Emtricitabine, Tenofovir_DF & Gonorrhea & 1009 \\
\hline AMP37 & M & $\geq 40$ & No & Cannabis & None & PrEP (2017) & Emtricitabine, Tenofovir_DF & None & 1454 \\
\hline AMV3 & M & $\geq 40$ & NO & NO & HIV (1996) & ART (1998) & Emtricitabine, Tenofovir_DF, Raltegravir & None & 382 \\
\hline AMV4 & M & $\geq 40$ & YES & Cannabis & HIV (2002) & ART (2002) & Emtricitabine, Tenofovir_AF, Bictegravir & None & 1172 \\
\hline AMV5 & M & $\geq 40$ & No & No & HIV (1995) & ART (2009) & Emtricitabine, Tenofovir_DF & None & 606 \\
\hline AMV8 & M & $\geq 40$ & No & NO & HIV (2002) & ART (2013) & Emtricitabine, Tenofovir_DF, Rilpivirine & None & 533 \\
\hline AMV9 & M & $\geq 40$ & YES & Cannabis & HIV (2002) & ART (2004) & Emtricitabine, Tenofovir_DF, Raltegravir & None & 536 \\
\hline AMV10 & M & $\geq 40$ & NO & NO & HIV (1992) & ART (1996) & Etravirine, Darunavir, Dolutegravir, Ritonavir & None & 269 \\
\hline AMV11 & M & $\geq 40$ & YES & NO & HIV (1997) & ART (1999) & Emtricitabine, Tenofovir_DF, Rilpivirine & None & 439 \\
\hline AMV13 & $\mathrm{F}$ & $\geq 40$ & YES & Cannabis & HIV (1994) & ART (1994) & Emtricitabine, Tenofovir_DF, Rilpivirine, Atazanavir, Raltegravir & None & 716 \\
\hline AMV14 & M & $\geq 40$ & YES & Cannabis & HIV (1996) & ART (1997) & Abacavir, Lamivudine, Atazanavir, Ritonavir & None & 983 \\
\hline AMV15 & M & $\geq 40$ & No & No & HIV (2003) & ART (2004) & Abacavir, Dolutegravir, Lamivudine & None & 468 \\
\hline AMV16 & M & $\geq 40$ & YES & NO & HIV (1987) & ART (2002) & Emtricitabine, Tenofovir_AF, Cobicistat, Elvitegravir & None & 1005 \\
\hline AMV17 & M & $\geq 40$ & YES & Cannabis & HIV (2014) & ART (2015) & Tenofovir_DF, Dolutegravir, Rilpivirine & None & 1267 \\
\hline AMV18 & M & $\geq 40$ & YES & Cannabis & HIV (2016) & ART (2016) & Tenofovir_DF, Dolutegravir, Lamivudine & None & 989 \\
\hline AMV19 & M & $\geq 40$ & NO & Cannabis & HIV (2005) & ART (2006) & Abacavir, Dolutegravir, Lamivudine & None & 565 \\
\hline AMV20 & M & $\geq 40$ & No & Cannabis & HIV (1997) & ART (1997) & Emtricitabine, Tenofovir_DF, Cobiscistat, Darunavir & None & 727 \\
\hline AMV21 & M & $\geq 40$ & YES & Cannabis & HIV (2012) & ART (2012) & Emtricitabine, Tenofovir_DF, Dolutegravir & None & 650 \\
\hline AMV23 & M & $<40$ & YES & Cannabis & HIV (2017) & ART (2017) & Emtricitabine, Tenofovir_AF, Cobicistat, Elvitegravir & None & 633 \\
\hline AMV25 & M & $\geq 40$ & No & NO & HIV (2006) & ART (2007) & Emtricitabine, Tenofovir_DF, Efavirenz & None & 906 \\
\hline AMV26 & M & $\geq 40$ & NO & No & $\operatorname{HIV}(2000)$ & ART (2010) & Emtricitabine, Tenofovir_DF, Efavirenz & None & 471 \\
\hline AMV27 & $\mathrm{M}$ & $\geq 40$ & No & No & HIV (2017) & ART (2017) & Emtricitabine, Tenofovir_AF, Raltegravir & None & 501 \\
\hline
\end{tabular}

ART, Antiretroviral Therapy; HTN, Hypertension; T1DM, Type 1 Diabetes Mellitus; T2DM, Type 2 Diabetes Mellitus; ADHD, attention deficithyperactivity disorder 
Table 2. Motif enrichment analysis for ATAC-seq and H3K27ac.

\begin{tabular}{|c|c|c|c|c|c|c|}
\hline Test & TFs & motif & $P$-value & FDR & $\begin{array}{l}\text { Target regions } \\
\text { with motif (\%) }^{a}\end{array}$ & $\begin{array}{c}\text { Background regions } \\
\text { with motif (\%) }^{b}\end{array}$ \\
\hline Open & ISRE & & $1.0 \mathrm{E}-12$ & $<1.0-5$ & $288(4.44 \%)$ & $1131(2.85 \%)$ \\
\hline Open & IRF2 & & $1.0 \mathrm{E}-07$ & $<1.0-5$ & $429(6.62 \%)$ & $2032(5.12 \%)$ \\
\hline Open & IRF1 & & $1.0 \mathrm{E}-05$ & $3.00 \mathrm{E}-04$ & $510(7.87 \%)$ & $2544(6.41 \%)$ \\
\hline Open & bHLHE40 & & $1.0 \mathrm{E}-05$ & $6.00 \mathrm{E}-04$ & $1038(16.01 \%)$ & $5587(14.07 \%)$ \\
\hline Open & NFkB-p50,p52 & & $1.0 \mathrm{E}-04$ & $1.10 \mathrm{E}-03$ & 706 (10.89\%) & $3702(9.33 \%)$ \\
\hline Open & Egr2 & & $1.0 \mathrm{E}-04$ & $1.60 \mathrm{E}-03$ & $1061(16.37 \%)$ & $5773(14.54 \%)$ \\
\hline Open & bZIP:IRF & & $1.0 \mathrm{E}-03$ & $3.12 \mathrm{E}-02$ & $887(13.68 \%)$ & $4888(12.31 \%)$ \\
\hline Open & Hoxb4 & & $1.0 \mathrm{E}-03$ & $3.26 \mathrm{E}-02$ & $396(6.11 \%)$ & $2059(5.19 \%)$ \\
\hline Open & C-Myc & & $1.0 \mathrm{E}-03$ & $3.91 \mathrm{E}-02$ & $1666(25.70 \%)$ & $9530(24.01 \%)$ \\
\hline Open & Slug & & $1.0 \mathrm{E}-03$ & 3.91E-02 & $1895(29.23 \%)$ & $10909(27.48 \%)$ \\
\hline Open & Oct4:Sox17 & & $1.0 \mathrm{E}-03$ & $3.91 \mathrm{E}-02$ & $252(3.89 \%)$ & $1262(3.18 \%)$ \\
\hline Open & REST-NRSF & & $1.0 \mathrm{E}-02$ & 4.17E-02 & $39(0.60 \%)$ & $140(0.35 \%)$ \\
\hline More Acetylated & ISRE & & $1.0 \mathrm{E}-66$ & $<1.0-5$ & $138(37.00 \%)$ & $3555(6.41 \%)$ \\
\hline More Acetylated & IRF1 & & $1.0 \mathrm{E}-50$ & $<1.0-5$ & $174(46.65 \%)$ & $7773(14.01 \%)$ \\
\hline More Acetylated & IRF2 & & $1.0 \mathrm{E}-50$ & $<1.0-5$ & $149(39.95 \%)$ & $5653(10.19 \%)$ \\
\hline More Acetylated & IRF3 & & $1.0 \mathrm{E}-46$ & $<1.0-5$ & $240(64.34 \%)$ & $15769(28.42 \%)$ \\
\hline More Acetylated & IRF8 & & $1.0 \mathrm{E}-36$ & $<1.0-5$ & $224(60.05 \%)$ & $15755(28.39 \%)$ \\
\hline More Acetylated & PU.1:IRF8 & & $1.0 \mathrm{E}-19$ & $<1.0-5$ & 149 (39.95\%) & $10687(19.26 \%)$ \\
\hline More Acetylated & T1ISRE & & $1.0 \mathrm{E}-12$ & $<1.0-5$ & $27(7.24 \%)$ & $680(1.23 \%)$ \\
\hline More Acetylated & IRF4 & & $1.0 \mathrm{E}-11$ & $<1.0-5$ & $173(46.38 \%)$ & $16446(29.64 \%)$ \\
\hline More Acetylated & PU.1-IRF & & $1.0 \mathrm{E}-07$ & $<1.0-5$ & $309(82.84 \%)$ & $39315(70.85 \%)$ \\
\hline
\end{tabular}

